\title{
Gelingende Kommunikation trotz Sprachbarrieren
}

\author{
Małgorzata Stanek
}

\begin{abstract}
Verständigungsschwierigkeiten zwischen einer Hebamme und der von ihr begleiteten Schwangeren, Gebärenden oder Wöchnerin erschweren die Betreuung. Im klinischen Umfeld haben Migrantinnen bisweilen das Gefühl, es werde von Fachpersonal mehr über sie geredet als mit ihnen. Dabei ist Dolmetschen - das Übersetzen von Gesprächen in Echtzeit - gerade in der gesundheitlichen Versorgung wichtig. Die Autorin fasst Regeln, Fallbeispiele und eine exemplarische Ablaufplanung für die transkulturelle Anamnese und Kommunikation zusammen.
\end{abstract}

\section{Hintergrund}

Mehr- und Fremdsprachlichkeit ist aus dem Berufsalltag der Hebammen nicht mehr wegzudenken. Vorbereitungskurse, Schwangerschaftskontrollen, Anamneseund Aufklärungsgespräche erfordern heute weit größere sprachliche und kulturelle Kompetenz als noch vor 30 Jahren. Der Einsatz von interkulturellen Dolmetscherinnen und Dolmetschern kann sich als eine unabdingbare Hilfe erweisen, ist jedoch gesetzlich nicht reguliert. Die Hürden liegen bei der Finanzierung der zusätzlichen Leistung durch die Krankenkassen sowie bei der Qualifizierung und Organisation der Dolmetschenden.

Ist die Finanzierung institutionell nicht gewährleistet kommen in erster Linie Familienangehörige, Freunde, Bekannte und häufig die eigenen Kinder zum Einsatz. Dies geschieht mit zum Teil verheerenden Konsequenzen, wenn Teile des Gesprächs nicht oder falsch übersetzt werden. Fürs Fachpersonal endet ein Gespräch ohne gegenseitige sprachliche Verständigung oftmals mit der Frage, ob der Patientin wirklich geholfen wurde.

\section{Von fehlender Verständigung zum Behandlungsfehler}

In Anlehnung an die im Grundgesetz verankerten Grundrechte auf rechtliches Gehör und ein faires Verfahren sollte der Anspruch auf wahrheitsgetreue Verdolmetschung auch im Gesundheits-, Bildungs- und Sozialwesen gesetzlich gesichert sein [11]. Die Beauftragte für Integration der Solothurner Spitäler brachte es gut auf den Punkt: „Es gibt genaue Vorschriften, wann und wie ein Verband gewechselt werden muss, und gleichzeitig ist eine so zentrale Frage wie die sprachliche Verständigung völliger Beliebigkeit überlassen. (...) Ein eklatanter Missstand.“ [8]
Gehrig und Graf (2009) stellten in ihrer Vorstudie zum Thema „Kosten und Nutzen des interkulturellen Übersetzens im Gesundheitswesen “ fest, dass Verständigungsprobleme und fehlende Sicherung der Kommunikation zwischen Gesundheitsfachperson und Patientinnen bzw. Patienten zu einer medizinischen Unter- oder Überversorgung führen können, die in beiden Fällen in höheren Behandlungskosten resultieren, erstere durch längere Krankheitsverläufe [3].

Die Übersetzung der Aufklärung über eine medizinische Behandlung zu verweigern, verhindert einen Informed consent, d. h. dass die betreute Frau bzw. Patientin ihre Einwilligung wohlüberlegt nach Kenntnisnahme aller relevanten Informationen abgeben kann (siehe hierzu auch Fachartikel von Matthias Diefenbacher in dieser Ausgabe DIE HEBAMME [2]). Somit ist die Verweigerung der Übersetzung, wo diese nötig wäre, weder juristisch noch ethisch haltbar [3]. Wer seine Sorgfaltspflicht gegenüber der Patientin erfülle, dürfe im Umkehrschluss keine Gespräche ohne zertifizierte Dolmetschende führen. Denn faktisch finde kein medizinisches Aufklärungsgespräch statt, wenn ein Betroffener dabei die Aussagen der Fachperson nicht versteht, argumentieren Gehrig und Graf weiter. Die fehlende Übersetzung bzw. Verständigung könne demnach als Behandlungsfehler konstatiert werden [3].

\section{Fallbeispiel}

Welche emotionalen und finanziellen Folgen solch ein Behandlungsfehler in der Praxis haben kann, zeigt folgendes Fallbeispiel:

Ein 16-jähriges Mädchen aus dem Kosovo kommt im Rahmen des Familiennachzugs in die Schweiz, wo sie 
kurz nach ihrer Ankunft an akuter Leukämie erkrankt. Als einzige Behandlung wird die Knochenmarktransplantation erwogen. Ihre Schwester kommt als Spenderin in Betracht. Ein spitalinterner Mitarbeiter übersetzt alle Gespräche. Die Ärzte erklären Diagnose, Prognose und Therapiemöglichkeiten sowie deren Risiken. Dazu zählt u. a. die Unfruchtbarkeit als Folge der Transplantation. Dieses Risiko übersetzt der Dolmetscher nicht - was keiner der Beteiligten (Ärzte und Familie) bemerkt.

Das Mädchen willigt in die Therapie ein. Bei der Ganzkörperbestrahlung wird der Heranwachsenden selbst bewusst, dass sie in deren Folge unfruchtbar werden könnte. Sie stellt Fragen, die aus Sicht des Personals bereits geklärt sind. Als sie versteht, dass sie nach der Behandlung kinderlos bleiben würde, bricht sie die Therapie ab und entscheidet sich gegen eine Knochenmarktransplantation. Grund: Sie sieht ohne die Möglichkeit auf einen erfüllten Kinderwunsch keinen Sinn im Leben.

Dieses Beispiel veranschaulicht die drastischen Folgen einer Versorgung, in der die sprachliche Verständigung zwischen Arzt und Patienten nicht gesetzlich geregelt ist und die nötigen Übersetzungsdienste nicht über die Krankenkasse abgerechnet werden können.

\section{STUDIE}

\section{Folgen unqualifizierten Dolmetschens}

In der Schweiz wurden 2019 erstmals zwei parlamentarische Vorstöße zur Finanzierung von interkulturellem Dolmetschen im ambulanten Bereich an den Bundesrat gestellt. Hintergrund waren Studien, die belegen, welche fatalen Auswirkungen der Einbezug von nicht qualifizierten Dolmetschern haben kann. 2019 wurde in einer Studie von Jäger et al bekannt, dass 60-70\% der Haus- und Kinderärztinnen bzw. -ärzte in der Schweiz den Eindruck haben, dass sie aufgrund von Sprachbarrieren keine qualitativ hochwertige Leistung erbringen können. Den Hauptgrund sehen sie im fehlenden Wissen, wie man Dolmetschende organisiert (30\%), in organisatorischen Aspekten (26\%) und in der fehlenden Finanzierung (25\%). Als weitere Gründe geben knapp $60 \%$ der Haus- und Kinderärztinnen und -ärzte an, dass die Organisation der Dolmetschenden zu umständlich ist. Rund 50 \% führen die fehlende Finanzierung als weiteren Grund an. Die Autoren kommen zum Schluss, dass der Einsatz professioneller Dolmetscher durch Sicherstellung der Finanzierung und Bekanntmachung des Angebots verbessert werden kann und dass bereits Best-practice-Beispiele (im Bereich der Dolmetscherdienste für Asylbewerber und Flüchtlinge) etabliert sind, auf die zurückgegriffen werden kann [4][6].

\section{Dolmetschen der Anamnese- und Therapiegespräche}

So lange es keine Vorgaben für die Übersetzung von Anamnese- und Therapiegesprächen gibt, ist die Frage: Wer kommt zum Einsatz? Nachfolgend seien die potenziellen Ressourcen und ihr Einsatz in der Praxis erörtert.

\section{Einsatz von Laiendolmetschenden}

Laiendolmetschende - auch Ad-hoc-Dolmetschende genannt - werden spontan (ad-hoc) zum Dolmetschen herbeigezogen, ohne dass sie entsprechende Kompetenzen vorlegen müssen. Darunter fallen u. a. :

- Inhouse-Mitarbeitende

- Familienangehörige (z. B. die eigenen Kinder)

- Freunde der Familie

Kommen Mitarbeitende z.B. einer Klinik zum Einsatz, haben diese meist eine eigene Migrationsbiografie oder sie verfügen über anderweitig erworbene Sprachkenntnisse. In der Regel wird auf Personal zurückgegriffen, das über eine Ausbildung im jeweiligen Fachbereich verfügt, um eine höhere Professionalität hinsichtlich der spezifischen Inhalte und Abläufe zu sichern.

\section{Einsatz zertifizierter Dolmetscher}

Unter interkulturell zertifizierten Dolmetschenden versteht man Menschen mit Migrationshintergrund, die sich in teils niederschwelligen Qualifikationsmaßnahmen entsprechend fortgebildet und qualifiziert haben. Neben ihrer Muttersprache bringen sie auch Kenntnisse von kulturellen und religiösen Hintergründen mit. Darin wird der Vorteil gesehen, dass sie ein kulturspezifisches Verständnis für persönliche Ängste und Vorbehalte haben. Interkulturell zertifizierte Dolmetschende sind u. a. :

- Sprach- und Kulturmittler

- Sprach- und Integrationsmittler

- Behördendolmetscher

- Krankenhausdolmetscher

Bezogen auf den D-A-CH-Raum kommen interkulturell zertifizierte Dolmetscher als Kulturvermittler (Deutschland), interkulturelle Dolmetschende und Vermittelnde (Schweiz) oder Kommunaldolmetschende (Österreich) zum Einsatz.

\section{STICHWORT}

Interkultureller Dolmetscher / Vermittler

Die schweizerische Interessengemeinschaft INTERPRET bietet mit dem eidgenössischen Fachausweis die Zertifizierung zum / zur Fachmann / frau interkulturelles Dolmetschen / Vermitteln auf Ebene des höheren Berufsabschlusses an. Das Konzept setzt auf das Gespräch im Trialog - einem Dialog zu dritt, 
bei dem der Dolmetschende eine „Verständigungsbrücke" zwischen einer oder mehreren Fachpersonen einerseits und Migrantinnen / Migranten andererseits herstellt. Dies geschieht unter Berücksichtigung des sozialen und kulturellen Hintergrunds der Gesprächsteilnehmenden. Dabei kann das Gespräch vor Ort sein oder der Dolmetscher wird via Telefon zugeschaltet. Trotz der „aktiven“ Rolle des Dolmetschenden, der als dritte Gesprächspartei verstanden wird, soll die Gesprächsleitung immer uneingeschränkt bei der Fachperson liegen [4].

\section{Hürden bei der Umsetzung in der Praxis}

\section{Finanzierung der Dolmetscherleistung}

Als Dozentin unterrichte ich seit Jahren u. a. Hebammen, Physiotherapeutinnen und Ergotherapeutinnen an der ZHAW im Weiterbildungsmodul „Transkulturelle Kompetenzen“. Die Rückmeldungen meiner Kursteilnehmerinnen zeigen, dass zwischen der Notwendigkeit, Dolmetscherdienste in Anspruch zu nehmen, und der praktischen Umsetzbarkeit noch eine Lücke klafft. So sei es für freiberufliche Hebammen bzw. Leistungserbringer nicht möglich, eine zertifizierte Dolmetschende für ein Gespräch vor Ort zu buchen oder einen Telefondolmetscherdienst zu nutzen, ohne die Kosten der betreuten Frau bzw. Patientin/Patient aufzubürden. Das ist vor allem für Menschen mit kleinem Einkommen oder für Sozialleistungsempfänger nicht zu leisten, aber auch bei Durchschnittsverdienern könne man diese Bereitschaft nicht voraussetzen: Für das Telefondolmetschen würden z. B. in der Schweiz $4 \mathrm{CHF}$ pro Minute anfallen, bei einer Mindestpauschale von 40 CHF.

\section{Erhöhter organisatorischer Aufwand und Leistungsdruck}

Angestellte Hebammen aus größeren Einrichtungen und Kliniken berichten, dass sie zwar auf ein beschränktes Angebot an Dolmetschern zurückgreifen können, solange finanzielle Mittel verfügbar sind. Der Einsatz der Dolmetschenden sei jedoch mit einem erhöhten Aufwand verbunden, der sie entsprechend Zeit koste. Das Dolmetschen eines Anamnese- oder Therapiegespräches ist derzeit in den Fallpauschalen zeitlich nicht berücksichtigt und bleibt somit bei der Zeiteinplanung vollkommen unberücksichtigt. Auch ist es bisher keine Krankenkassenleistung.

In der Folge berichten die von mir befragten Hebammen und sonstigen Leistungserbringer von steigendem Druck: Dem zusätzlichen organisatorischen Zeitaufwand stehen knappe Zeitressourcen und die gleichzeitig hohen Anforderungen an ihre Arbeit gegenüber (u. a. allgemeine Vorschriften, interne Weisungen des
Arbeitgebers, Checklisten, Vorgaben durch QS-Maßnahmen, berufsethische Standards, eigener Anspruch). Viele greifen nach eigenen Angaben daher nur dann auf externe Dolmetschende zurück, wenn sie tatsächlich müssten. Das ist insbesondere der Fall bei komplexen und sehr komplexen Inhalten oder wenn die Familie bzw. Freunde, die zum Dolmetschen mitkommen, nicht mehr weiterhelfen können.

Merke

Gedolmetschte Arzt-Patienten-Gespräche sind mit erhöhtem Zeitaufwand bei gleichzeitig teilweise sehr knappen Zeitressourcen verbunden. Den Fachpersonen fehlt es an finanziellen und zeitlichen Ressourcen, aber auch an Wissen und Schulungen zur effizienten Führung von interkulturellen Gesprächen.

\section{Ein Weg aus dem Dilemma}

Wie aber kann die Kommunikation mit der betreuten Frau oder Familie trotz Sprachbarrieren und begrenzter Ressourcen gelingen? Und was beeinflusst Fachpersonen in ihrer Entscheidung, ob sie eine Mittelsperson hinzuzuziehen? „Es braucht den aktiven Willen der Fachperson, (a) überhaupt jemanden beziehungsweise eine bereits bekannte Person anzufordern und (b) allenfalls den Einsatz der Mittelsperson an Bedingungen zu knüpfen (Ausbildung, Vorbereitung, Konditionen Einsatz, usw.).“ [9] Das ist eines der Ergebnisse der Studie „Interkulturelle Übersetzung und Vermittlung“ der ZHAW, die Gesprächssituationen zwischen Fachpersonen und nicht deutschsprachigen Klientinnen und Klienten im Sozial- und Bildungsbereich im Kanton Zürich untersuchte.

In seiner Studienzusammenfassung schreibt Thomas Redmann, zur Verminderung von Missverständnissen, Misstrauen und Konflikten müssten verschiedene Formen von Ressourcen zur Verfügung stehen. Insbesondere sei bei den Ressourcen und transkulturellen Kompetenzen von Fachpersonen anzusetzen: „Diese können durch Sensibilisierung und Weiterbildung darin unterstützt werden, interkulturell vermittelte Gespräche auf Grundlage eines spezifischen Wissens und einer reflektierten Haltung vorzubereiten, weiterzuentwickeln und zum Erfolg zu führen." [9]

Meine Gespräche mit Hebammen und anderen Leistungserbringern zeigen, dass diese ein großes Interesse haben, dass ihre Gespräche mit den von ihnen betreuten Frauen, Familien, Klientinnen und Klienten bzw. Patientinnen und Patienten gewinnbringend für alle Beteiligten verlaufen und dass die Problemlösung in Zusammenarbeit mit dem Leistungsempfänger gelingt. Nachfolgend seien einige Grundlagen der interkulturellen Anamnese und Therapiegespräche zusammengefasst. 


\section{DEFINITION}

Dolmetschen vs. Übersetzen

- Dolmetschen ist die mündliche Übertragung eines einmalig (in der Regel mündlich) vorgetragenen Textes in einen aus Zeitmangel kaum korrigierbaren Text der Zielsprache.

- Übersetzen ist die schriftliche Übersetzung eines Ausgangstextes in einen wiederholt korrigierbaren Text der Zielsprache.

(nach Otto Kade [7])

\section{Sprachbarrieren überwinden}

Lange Zeit hat sich der Mythos gehalten, „eine gute Übersetzung“ sei eine Eins-zu-Eins-Übertragung oder wörtliche Übertragung. Aus translationswissenschaftlicher Sicht ist dieses Verständnis von sprachlicher Übertragung überholt. Um es mit einfachen Worten zu sagen: Wir dolmetschen keine Worte, sondern Sinneinheiten. Eine sinngemäße Übertragung findet unter Berücksichtigung der kontextuellen Aspekte einer Äußerung statt und hat die optimale Vermittlung des Gemeinten zum Ziel.

\section{Grundregeln für die Sprachübertragung}

Bei der Übertragung gelten drei goldene Regeln:

1. nichts auslassen

2. nichts hinzufügen

3. keine Inhalte verändern!

„Übersetze so getreu wie möglich und so frei wie nötig.“ Dieses Postulat trifft die Ansprüche an die richtige Vorgehensweise beim Sprachtransfer von Sprache A nach B am gezieltesten. Aus berufsethischer Sicht ist auf die „Vollständigkeit und Genauigkeit“ zu achten. Diese Regeln einzuhalten, ist unabdingbar für Leistungserbringer der Gesundheitsversorgung, die als Gesprächsleiter das

-Tab. 1 Anpassung des Sprachregisters an das des Patienten.

\begin{tabular}{|l|l|l|}
\hline $\begin{array}{l}\text { Aussage Arzt bzw. } \\
\text { Fachperson }\end{array}$ & $\begin{array}{l}\text { Übertragung } \\
\text { Dolmetscher }\end{array}$ & $\begin{array}{l}\text { Medizinischer Laie } \\
\text { bzw. Patient mit } \\
\text { geringem } \\
\text { Bildungsstand }\end{array}$ \\
\hline Analgetika & übersetzt „Analgetika“ & $\begin{array}{l}\text { bleibt außen vor, beide } \\
\text { fachsimpeln }\end{array}$ \\
\hline Analgetika & $\begin{array}{l}\text { übersetzt } \\
\text {,Schmerzmittel“ }\end{array}$ & $\begin{array}{l}\text { versteht das Gesagte } \\
\text { - Sprachregister- } \\
\text { wechsel durch den } \\
\text { Dolmetschenden }\end{array}$ \\
\hline Schmerzmittel & übersetzt & $\begin{array}{l}\text { versteht das Gesagte - } \\
\text { optimale Situation }\end{array}$ \\
\hline Quelle: Eigene Darstellung & \\
\hline
\end{tabular}

Gespräch mit Klienten bzw. Patienten steuern und führen müssen.

Ein wichtiger Aspekt der sprachlichen Übertragung ist die Anpassung des Sprachregisters an jenes des Patienten ( $\triangleright$ Tab. 1). Idealerweise sorgt für diesen Sprachregisterwechsel die Fachperson und nicht der Dolmetscher, um Fehlübersetzungen zu vermeiden.

Merke

Auch ein Dolmetscher - ob Laiendolmetscher oder zertifizierter Dolmetscher - kann nur übertragen, was er oder sie verstanden hat. Daher ist für eine gelingende Kommunikation über Sprachbarrieren hinweg wichtig, dass sich der Leistungserbringer verständlich ausdrückt bzw. der „leichten Sprache“ bedient.

\section{Die Rolle des Dolmetschenden}

Die Hauptaufgabe des Dolmetschenden ist es, die Verständigung zwischen zwei Parteien herzustellen und für gelingende Kommunikation zu sorgen, ganz unabhängig von ihrem Inhalt. Sollte jemand zum Beispiel beabsichtigen, einen Streit loszutreten oder seine Missbilligung seinem Gesprächspartner gegenüber zum Ausdruck zu bringen, so ist die Übersetzung als gut zu erachten, wenn ihm das mit der übersetzten Aussage tatsächlich gelungen ist bzw. zumindest die gleichen Voraussetzungen für eine Reaktion des Gegenübers, wie in der Ausgangssprache der Fall war, gegeben sind.

\section{Vertrauensaufbau als Schlüssel zur gelingenden Kommunikation}

Nun ist die Frage, wie der Vertrauensaufbau gelingt, wenn die Patientinnen nicht die Landesprache oder über genügend Englischkenntnisse verfügen. Und wie können Leistungserbringer die Qualität gedolmetschter Gespräche verbessern? Neben einer empathischen Grundhaltung gibt es weitere Mittel, die eine wirksame Gesprächsführung unterstützen.

CHECKLISTE

Mittel für eine wirksame Gesprächsführung

- Empathische Grundhaltung einnehmen

- Augenkontakt herstellen

- Vorwissen erfragen

- Aktiv zuhören und Fragen stellen

- Langsam und deutlich sprechen

- Alltagssprache verwenden

- Kurze und einfache Sätze bilden

- Zahl der Botschaften begrenzen

- Informationen in «Häppchen» aufteilen

- Handlungsanleitungen geben

- Wichtige Punkte betonen und wiederholen 
- Visuelle Medien einsetzen

- Schriftliche Informationen bereitstellen

- Rückfragen stellen, das Gespräch zusammenfassen lassen

- Nächste Schritte erklären

- Patienten ermutigen, Fragen zu stellen

\section{Die Gesprächsführung liegt bei der Hebamme}

Eine der wichtigsten Maßnahmen ist das Vorgespräch zwischen Hebamme bzw. Leistungserbringer und interkulturell Dolmetschendem. Wenn Angehörige oder Freunde diese Rolle übernehmen, sind sie idealerweise zu instruieren, dass sie jetzt in der Rolle des Dolmetschenden und z.B. nicht in der des Ehemanns oder der Freundin übersetzen. Das Vorgespräch sollte von der Hebamme angeboten werden. Darin ist auch deutlich zu machen, dass die Gesprächsleitung bei der Hebamme liegt.

Wichtig ist zudem, sich die Situation der Gesprächspartnerin bewusst zu machen: Je mehr die Hebamme die Herkunft und das Bildungsniveau der betreuten Frau in ihrer Formulierung berücksichtigt, desto weniger muss der Dolmetschende entscheiden, inwieweit das Gesagte an die kulturellen Normen und kognitiven Möglichkeiten des Gegenübers angepasst werden muss. Jedes selbstständige Entscheiden des Dolmetschenden birgt die Gefahr, dass dieser den Sinn der Aussage durch eigenständige Formulierungen und Erklärungen modifiziert und seine Rolle als aktiver Gesprächsteilnehmer missversteht und schließlich sogar die Gesprächsführung übernimmt. Das gilt besonders vor dem Hintergrund, dass viele dolmetschende Personen nicht oder nur niederschwelig ausgebildet sind. Eigenständige Aussagen des Dolmetschenden verunsichern die betreute Frau bzw. Patientin - sie weiß nicht, zu wem sie sprechen soll. Die Tendenz ist vorhanden, dass die Dolmetscherin von Patienten direkt angesprochen wird. Das entmachtet den Leistungsträger in seiner Rolle als Gesprächsführer zusätzlich.

Bischoff (2000) plädiert dafür, dass der für den Gesprächsverlauf verantwortliche Leistungserbringer sowohl dem Patienten als auch der Dolmetscherin eine gewisse Freiheit zugestehen sollte. Dabei sollte er aber stets dafür sorgen, dass er dem Gespräch folgen kann und dass sich das Gespräch nicht verlagert, sodass es im weiteren Verlauf nur noch zwischen Patient und Dolmetscherin bzw. zwischen Leistungserbringer und Dolmetscherin stattfindet. Damit ist der berufsethische Standard der Transparenz gesichert. Derartige Situationen, die einen der Gesprächsteilnehmer ausschließen, sollten unbedingt vermieden werden [1]. 


\section{Kurzanleitung für zu dolmetschende Gespräche}

Im Rahmen des KTI-Projektes „Anforderungs- und Rollenprofil für Dolmetschende im medizinischen Bereich“ wurde an der ZHAW eine „Kurzanleitung für erfolgreiche Gespräche“ erarbeitet und durch die KTI (Kommission für Technologie und Innovation) mitfinanziert [10]. Aus den Tipps für medizinische Fachpersonen lässt sich auch Handlungswissen für die Hebammenarbeit ableiten:

\section{Vor dem Gespräch}

- Klären Sie vor dem Gespräch, welche Fachbegriffe Sie benutzen (z. B. Dopton), ob es sich in „leichter Sprache“ sagen lässt (z. B. Herztonmesser) und ob die Dolmetschende ein Äquivalent in der Muttersprache der Frau kennt.

- Informieren Sie die Dolmetschende über Inhalt, Ziel und Dauer des Gesprächs.

- Weisen Sie die Dolmetschende darauf hin, dass sie das Gespräch getreu wiedergeben muss, keine eigenen Interpretationen, Erklärungen oder Sonstiges hinzufügen darf.

- Fordern Sie die Dolmetschende auf, bei Ihnen nachzufragen, wenn sie etwas nicht verstanden hat.

\section{Während des Gesprächs}

- Stellen Sie Dolmetschende und Patientin einander namentlich vor.

- Erklären Sie der Frau bzw. Familie, dass der oder die Dolmetschende unter Schweigepflicht steht und alle Gesprächsinhalte vollständig wiedergibt.

- Halten Sie Augenkontakt mit der betreuten Frau und sprechen Sie diese immer direkt an, sprechen Sie nicht mit der Dolmetschenden in der dritten Person über die Frau (also nicht: „Welche Veränderungen hat sie bemerkt?“, sondern: „Welche Veränderungen haben Sie bemerkt?“)

- Formulieren Sie verständlich, klar und deutlich, in vollständigen Sätzen und vermeiden Sie Fachwörter.

- Fragen Sie bei der Patientin nach, wenn Sie das Gefühl haben, dass sie etwas nicht versteht.

- Bitten Sie die Patientin zusammenzufassen, was sie verstanden hat.

\section{Nach dem Gespräch}

- Vergewissern Sie sich bei der Dolmetschenden, ob die Frau ihrer Einschätzung nach alles verstanden hat und das Gespräch für sie korrekt war.

- Bitten Sie die oder den Dolmetscher um ein Feedback zu Ihrer Gesprächsführung.

\section{ZUSAMMENFASSUNG}

Regeln für Gespräche mit Dolmetschenden Bereiten Sie sich auf das Gespräch vor: Absprachen sind zu treffen bzgl.:

- Vorstellung: Stellt sich die Dolmetscherin selbst vor oder übernehmen Sie das als Gesprächsführerin?

- Einführungssätze: Weisen Sie die Dolmetschende im Beisein der Klientin auf Ihre Schweigepflicht hin (Vertrauensaufbau).

- Interaktion: Vereinbaren Sie mit der Dolmetscherin, dass sie sich im Gespräch zurückhält und nur dann (z. B. per Handzeichen) zu Wort meldet, wenn sie etwas nicht versteht - akustisch oder inhaltlich!

- Gesprächstempo: Vereinbaren Sie ein Handzeichen für den Fall, dass Sie zu schnell sprechen. So muss das Gespräch nicht unterbrochen werden.

- Goldene Regeln: Vermitteln Sie, worauf es beim Dolmetschen ankommt - nichts auslassen, nichts hinzufügen, nichts verändern.

- Gesprächsführung: Suchen Sie immer das Gespräch mit der Dolmetscherin, äußern Sie Ihre Ziele und Erwartungen an die dolmetschende Person explizit und behalten Sie die Gesprächsführung.
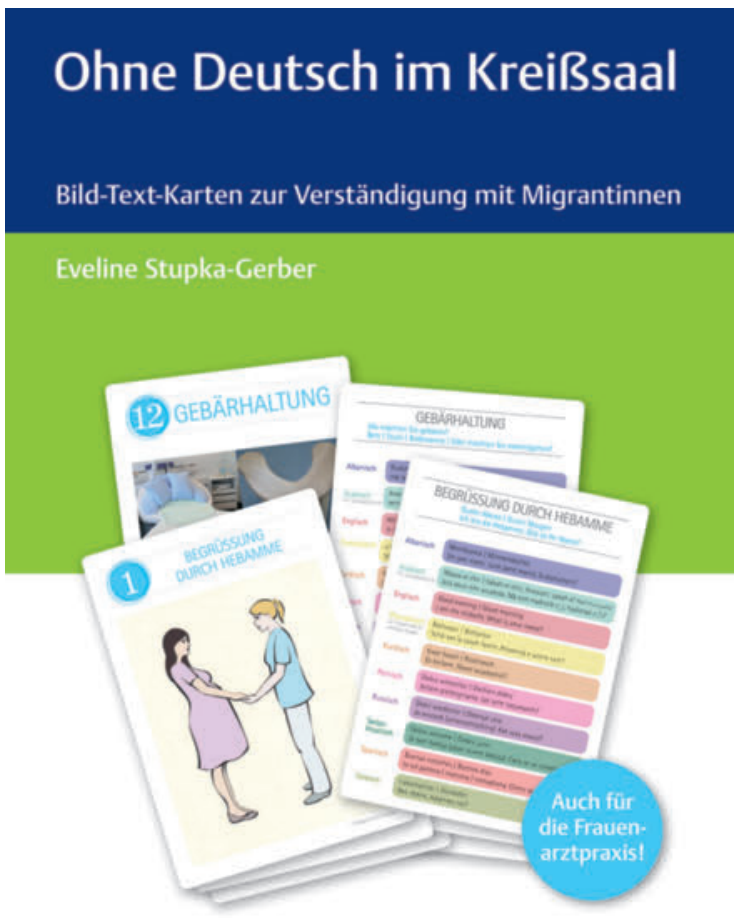

Hippokrates

Auch mithilfe von Bildern lassen sich bei Sprachbarrieren geburtshilfliche Sachverhalte leichter erklären. (Covergestaltung: Thieme Gruppe) 
- Grenzen setzen: Weigern Sie sich, in kritischen Situationen Familienangehörige dolmetschen zu lassen!

- Vorbereitung ermöglichen: Für sich selbst und die Dolmetscherin. Planen Sie mehr Zeit für die Durchführung von gedolmetschten Gesprächen ein und informieren Sie die Dolmetschende vorab, um welche Fachtermini und um welche Art des Gesprächs es sich handeln wird. So ermöglichen Sie der Dolmetscherin, sich vorzubereiten.

\section{Handlungsoptionen des Dolmetschenden berücksichtigen}

Ausgebildete Dolmetschende, die mit berufsethischen Standards vertraut sind, werden sich bei Rückfragen an die Fachperson wenden, bevor sie etwas übersetzen, bei dem sie noch Erklärungsbedarf haben. Weil aber nicht immer ein zertifizierter Dolmetschender zur Verfügung steht, ist es wichtig, dass die Fachperson genau beobachtet, wie die Übertragung des Gesprächs abläuft. So lassen sich Fehlübertragungen rechtzeitig erkennen und vermeiden, z.B. weil der übersetzende Partner oder nahe Verwandte der Frau religiös oder kulturell bedingte Besonderheiten und Gepflogenheiten in seine Übersetzung einfließen lässt.

- Sagt er etwas, um das Gesicht zu wahren, ohne Berücksichtigung des Gesagten oder Gemeinten?

- Übersetzt er nur einen Bruchteil und lässt dabei versehentlich oder vorsätzlich wichtige Inhalte aus?

- Fügt er bei Erklärungsversuchen zusätzliche Inhalte hinzu, die in der Äußerung weder gesagt noch gemeint wurden?

- Fällt es ihm schwer vor allen Beteiligten sein Unwissen zuzugeben (Abhängig von Kultur und Geschlecht kann dies als Gesichtsverlust wahrgenommen werden) und die Fachpersonen um Erklärung des Gesagten zu bitten?

- Wenn diese Fragen mit einem „Ja“ beantwortet werden, sollte die Hebamme eine zertifizierte Dolmetscherin bestellen und auf das Geschlecht achten. Den Vermittlungsstellen kann das Geschlecht genannt werden. In diesem intimem Bereich der Geburt sind männliche Dolmetschende keine Hilfe.

\section{Fazit}

Vor dem Hintergrund, dass der Einsatz externer, professioneller Dolmetscher im Gesundheitswesen nicht flächendeckend gesichert ist, und tendenziell sich noch immer viele Laiendolmetschende in der Praxis feststellen lassen, ist es unverzichtbar, dass die Hebammen bzw. Leistungserbringer im Gesundheitswesen auf die gedolmetschten Gespräche durch Schulungen umfangreich vorbereitet werden und sich ihrer Handlungs- und Steuerungsmöglichkeiten als Gesprächsleitende bewusst sind.

Trotz Richtlinien zu berufsethischen Prinzipien des Dolmetschenden, die sehr weit gefasst sind, variiert das Rollenverständnis der zertifizierten Dolmetschenden. Umso wichtiger ist es, dass das Vorgespräch auf Initiative des Leistungserbringers und damit Auftraggebers der Dolmetschenden vor dem Gespräch erfolgt. Ebenfalls liegt es in der Verantwortung des Gesprächsführers auf eine verständliche Sprache zu achten, medizinischen Fachjargon zu vermeiden, und den direkten Kontakt zum Patienten herzustellen. Beispiele aus der Praxis zeigen, dass die Fachkräfte bei größerer Zeiteinplanung für die gedolmetschten Gespräche und eingeplanter Vorbereitung größere Erfolge bei den Gesprächen erzielen.

Sowohl Leistungserbringer als auch zertifizierte Dolmetschende wünschen sich klare Regeln und Abläufe. Damit es im Hebammenalltag klappt, erstellen Sie sich eine individuelle Checkliste aus der „Kurzanleitung für erfolgreiche Gespräche“ für Ihre beruflichen Settings. 
Gedolmetschte Gespräche erfolgen kostensparsam, wenn sie effizient ablaufen und beide Gesprächsparteien das Gefühl haben, ihre Inhalte vermittelt zu haben und verstanden worden zu sein. So wird vermieden, dass bereits geklärte Fragen wiederkehren, die Leiden und Behandlungsdauer verlängert werden, Folgekrankheiten aufgrund von fehlender Kommunikation auftreten und höhere Behandlungskosten entstehen.

\section{FÜR DIE PRAXIS \\ Bild-Text-Karten für Gespräche mit \\ Migrantinnen}

Ein eigenständiges Tool für Hebammen, Gynäkologinnen und die Kommunikation im Kreißsaal sind die Bild-Text-Karten für die Verständigung mit Migrantinnen von Eveline Stupka-Gerber: Ohne Deutsch im Kreißsaal - Basics für die Kommunikation mit Migrantinnen in der Geburtshilfe. 2014, Hippokrates, 128 S., 50 Abb., ISBN:

9783830455523, 39,99 Euro.

Um Hebammen und Geburtshelferinnen in der interkulturellen Kommunikation zu stärken, hat Stupka 24 Bild-Textkarten mit zentralen Sätzen bei der Betreuung von Frauen in 15 Sprachen erarbeitet. Diese sollen eine Hilfestellung für die Hebammen sein, wenn sie nicht auf externe Dolmetschende zurückgreifen können. Darüber hinaus vermittelt die Autorin interkulturelles Hintergrundwissen, z.B. dass in manchen Religionen das Neugeborene unmittelbar nach der Geburt als unrein gilt. In diesen Fällen ist es wichtig, der Frau das blutige Neugeborene nicht reflektorisch auf die Brust zu legen, sondern zunächst zu fragen, ob das Kind zuerst gewaschen werden sollte. Dieses interkulturelle Wissen sei essenziell für eine gute Betreuung, so die Autorin. Die Fachperson kann es selbst erlernen oder unterstützend von Dolmetschenden darauf aufmerksam gemacht werden. Voraussetzung ist, dass allen Beteiligten ihre Rollen und Aufgaben bekannt sind.

\section{Autorinnen / Autoren}

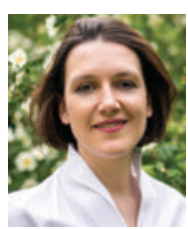

Małgorzata Stanek studierte Dolmetschen und Übersetzen für Polnisch und Englisch an der Humboldt Universität zu Berlin. Erfahrungen im kommunalen Dolmetschen sammelte sie während ihres Auslandsstudiums in Großbritannien als public service interpreter. Sie arbeitete als freiberufliche Übersetzerin und Dolmetscherin für die Polizeibehörden und Amtsgerichte in Berlin und Brandenburg und publizierte zur Problematik des Einsatzes unqualifizierter Dolmetscher. 2013 bis 2015 leitete sie die Weiterbildungskurse im Bereich Gerichts-, Behörden- und Spitaldolmetschen an der ZHAW,
Departement Linguistik, in Winterthur. Seitdem unterrichtet sie Transkulturelle Kompetenzen in der Hebammenweiterbildung an der ZHAW Gesundheit.

\section{Korrespondenzadresse}

E-Mail: info@transponere.com

Literatur

[1] Bischoff A, Loutan L. Mit anderen Worten - Dolmetschen in Behandlung, Beratung und Pflege. Genf: Département de Médecine Communautaire Hôpitaux Universitaires de Genève; 2000

[2] Diefenbacher M. Patientenaufklärung in der Hebammenarbeit. Die Hebamme 2020 (2): 54-63

[3] Gehrig M, Graf I. Kosten und Nutzen des interkulturellen Übersetzens im Gesundheitswesen (Vorstudie). Bern: Büro für arbeits- und sozialpolitische Studien BASS AG; 2009. Im Internet: https://nbn-resolving.org/urn:nbn:de:0168-ssoar377913

[4] INTERPRET. Verständigung im interkulturellen Kontext Begriffsklärungen. Stand 06.01.2020. Im Internet: https:// www.inter-pret.ch/de/angebote/begriffsklaerungen-39.html

[5] Jaeger FN, Pellaud N, Laville B et al. Barriers to and solutions for addressing insufficient professional interpreter use in primary healthcare. BMC Health Serv Res 2019; 19, 753. doi: org/10.1186/s12913-019-4628-6

[6] JaegerFN, Pellaud N, Laville B et al. The migration-related language barrier and professional interpreter use in primary health care in Switzerland. BMC Health Serv Res 2019; 19, 429. doi:org/10.1186/s12913-019-4164-4

[7] Kade O. Zufall und Gesetzmäßigkeiten in der Übersetzung. Leipzig: VEB Enzyklopädie; 1968

[8] Praz S. Wenn Kinder Krankheiten übersetzen (03.02.2014). Im Internet: https://www.beobachter.ch/gesundheit/medi zin-krankheit/gesundheitswesen-wenn-kinder-krankheiten-u bersetzen; Stand: 06.01.2020

[9] Redmann T. Interkulturelle Übersetzung und Vermittlung. Die Zusammenarbeit zwischen nicht deutschsprachigen Eltern, Mittelspersonen und Fachpersonen im Sozial-und Bildungsbereich. ZHAW, Soziale Arbeit, Forschung und Entwicklung (2009). Im Internet: https://www.zhaw.ch/storage/shared/sozialearbeit/For schung/Vielfalt_gesellschaftliche_Teilhabe/Migration/Interkultu relle_UEbersetzung_und_Vermittlung_Kurzbericht.pdf

[10] Sleptsova M. Patient - Dolmetscher - Fachperson. Anleitung für erfolgreiche Gespräche. ZHAW Institut für Übersetzen und Dolmetschen Weiterbildung, Winterthur

[11] Stanek M. Dolmetschen bei der Polizei. Zur Problematik des Einsatzes unqualifizierter Dolmetscher. Berlin: Frank \& Timme; 2011

\section{Bibliografie}

DOI https://doi.org/10.1055/a-1110-2055

Die Hebamme 2020; 33: 32-40

(c) Georg Thieme Verlag KG Stuttgart · New York ISSN 0932-8122 